This is a self-archived - parallel published version of this article in the publication archive of the University of Vaasa. It might differ from the original.

\title{
Peer-to-peer energy trading between wind power producer and demand response aggregators for scheduling joint energy and reserve
}

Author(s): Vahedipour-Dahraie, Mostafa; Rashidizadeh-Kermani, Homa; Shafiekhah, Miadreza; Siano, Pierluigi

Title: $\quad$ Peer-to-peer energy trading between wind power producer and demand response aggregators for scheduling joint energy and reserve

Year: $\quad 2020$

Version: Final draft (post print, aam, accepted manuscript)

Copyright (C)2020 IEEE. Personal use of this material is permitted. Permission from IEEE must be obtained for all other uses, in any current or future media, including reprinting/republishing this material for advertising or promotional purposes, creating new collective works, for resale or redistribution to servers or lists, or reuse of any copyrighted component of this work in other works.

\section{Please cite the original version:}

Vahedipour-Dahraie, M., Rashidizadeh-Kermani, H., Shafie-Khah, M., \& Siano, P., (2020). Peer-to-peer energy trading between wind power producer and demand response aggregators for scheduling joint energy and reserve. In: IEEE Systems Journal. https://doi.org/10.1109/ JSYST.2020.2983101. 


\title{
Peer-to-Peer Energy Trading between Wind Power Producer and Demand Response Aggregators for Scheduling Joint Energy and Reserve
}

\author{
Mostafa Vahedipour-Dahraie, Homa Rashidizadeh-Kermani, Miadreza Shafie-khah, Senior Member, \\ IEEE, and Pierluigi Siano, Senior Member, IEEE
}

\begin{abstract}
In this paper, a stochastic decision making framework is presented in which a wind power producer (WPP) provides some required reserve capacity from demand response aggregators (DRAs) in a peer-to-peer (P2P) structure. In this structure, each DRA is able to choose the most competitive WPP, and purchase energy and sell reserve capacity to that WPP under a bilateral contract-based P2P electricity trading mechanism. Based on this structure, the WPP can determine the optimal buying reserve from DRAs to offset part of wind power deviation. The proposed framework is formulated as a bi-level stochastic model in which the upper level maximizes the WPP's profit based on the optimal bidding in the day-ahead and balancing markets, whereas the lower level minimizes DRAs' costs. In order to incorporate the risk associated with the WPP's decisions and to assess the effect of scheduling reserves on the profit variability, conditional value-at-risk (CVaR) is employed.
\end{abstract}

Index Terms-Demand response, electricity market, reserve, peer-to-peer, wind power.

\section{NOMENCLATURE}

\section{A. Sets and indices}

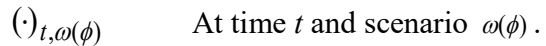

$(\cdot), \overline{(\cdot)} \quad$ Symbols for variable lower and upper limits.

$t(T) \quad$ Index (set) of time periods.

$\phi(\Phi) \quad$ Index (set) of scenarios of rivals' offering prices.

$\omega(\Omega) \quad$ Scenario index (set) of market prices and total loads.

$W_{P p}, W_{p} p^{\prime} \quad$ Index of wind power producer (WPP).

$N_{W p p} \quad$ Set of WPPs.

\section{B. Parameters}

$E_{t, \omega}^{D} \quad$ Total demand of DRAs (MWh).

$\widehat{E}_{t}^{D} \quad$ Expected demand of DRAs (MWh).

$E_{t, \omega}^{N R L} \quad$ Total demand of non-responsive loads (MWh)

$E_{t, \omega}^{\text {wind }} \quad$ Wind energy generation (MWh).

$X_{W p p t, \phi}^{\text {Init }} \quad$ Percentage of responsive loads supplied by each $X_{W p p, t, \phi}^{\text {Init }} \quad$ WPP, initially.

$\lambda_{t, \omega}^{B^{+}\left(B^{-}\right)}$Positive (negative) balancing market price ( $€ / \mathrm{MWh})$.

$\lambda_{t p}^{C_{u p}}\left(\lambda_{t}^{C_{d n}}\right) \quad$ Price of up and down reserve capacity allocated by DRAs (€/MWh).

$\lambda_{t, \omega}^{D A, S e l l}, \quad$ Selling (buying) day-ahead (DA) market prices

$\left(\lambda_{t, \omega}^{D A, B u}\right)$

$\lambda_{t, \phi}^{W p p}$

Offering price by rival WPPs (€/MWh).

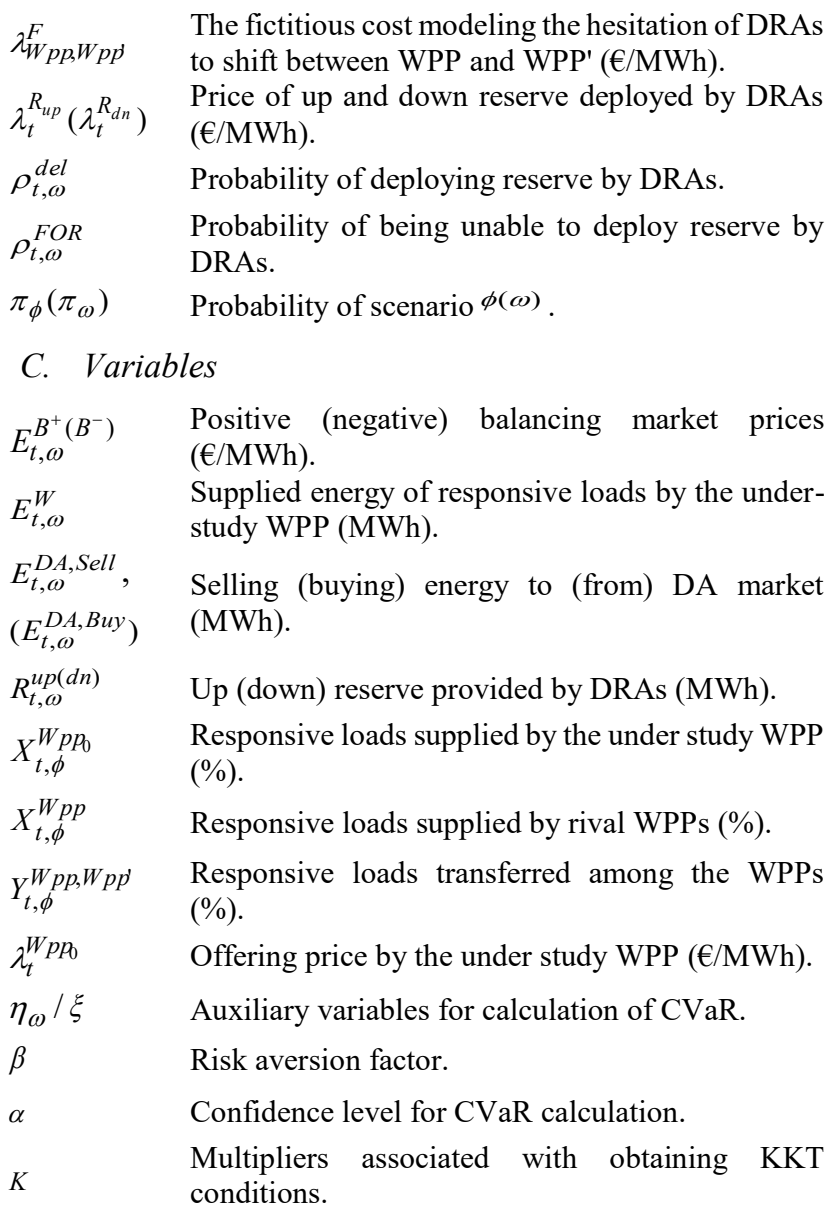

\section{INTRODUCTION}

When increasing the penetration of wind generating in the power system, the volatility of supply-side increases thus leading to significant challenges in the energy management system [1]. In such a condition, an extra flexibility requirement that is commonly fulfilled by additional operation reserves is needed to guarantee the system's security and reliability [2]. When the penetration of wind power increases, wind power producers (WPPs) should be more responsible for the deviation of their wind power production from the forecasted value. The findings of previous researches show that compensation of wind power deviations in the balancing market is costly for the WPPs. For example, in Spain, the WPP's revenue loss from deviation penalties is estimated to be up to $10 \%$ of the total revenue [3]. By applying strategic bidding in the electricity market, the WPP's revenue loss associated with wind power variability 
would be mitigated, significantly [4]. In this regard, authors in [5] have presented a two-stage stochastic optimization model to determine optimal bids that control the risks related to profit variability of a WPP. In [6], a joint day-ahead (DA) energy and reserve auction is studied, where producers offer their conventional power strategically based on a complementarity approach and their wind power at generation cost based on a forecast. Moreover, some comprehensive optimal bidding strategies have been proposed to consider the imbalance penalty prices in the WPP's decision making strategy [7]. The profit sharing problem for a group of WPPs which jointly offer energy in a well-known fully fledged multimarket trading floor model including the DA market, the adjustment market, and the real time energy balancing market are investigated in [8].

Careful review of prior works shows that many researchers have focused on optimal bidding of wind power in a conventional two-settlement energy trading structure including a DA and real-time markets. In such a structure, the system operator is responsible of scheduling and deploying reserves for wind power deviations, and the WPPs only submit energy offers in DA market encountering with penalty for the submitted power deviations [9]. A stochastic optimization model has been presented in [10] to determine the best offering prices for a hybrid power plant consisting of a WPP and a demand response (DR) aggregator in the power market. DR is used to cover the uncertainty of wind power and mitigate the imbalance cost. In [11], load profile attributes such as utilization and DR load availability/ flexibility is incorporated into DR (seller) bid model thus into DR exchange market clearing with the consideration of customer behavior. Moreover, a real-time decentralized demand-side management is proposed in [12] to adjust the real-time residential load to follow a preplanned DA energy generation by the microgrid, based on predicted customers' aggregate load. In that study, customers exploit renewable energy and energy storage systems and decide optimal strategies for their charging/discharging, taking into account their operational constraints.

Nevertheless, few research works have studied optimal offering strategies of WPPs in order to schedule both the energy and the reserve from the WPPs viewpoint. In [13], an optimal bidding strategy has been presented from the WPP perspective, in which the optimal amount of the purchased reserve and biding energy in the DA market is determined. In that strategy, a WPP commits some of its own reserve to offset its power deviations, instead of entirely being penalized in the ex-post imbalance market. However, finding of the previous works show that when WPPs participate in the reserve market, their revenues increase if compared with the case when they pay the imbalance prices in the real-time market [13]. Although, in recent years different scheduling strategies have been presented for determining reserve required by WPPs, almost, all of these approaches focus on the reserve requirement from the system operator perspective. By developing smart grids in recent years, it is possible for WPPs to improve their revenue by effectively utilizing various resources such as responsive loads. In fact, WPPs can use less expensive reserve capacities from DR to mitigate the uncertainty of wind generation [14]. In this regard, in [15], a bi-level market model has been presented for a windintegrated electricity market, where DR programs are implemented to deal with wind variability. A stochastic model from a microgrid operator perspective is investigated in [16] for energy and reserve scheduling considering risk management strategy. A stochastic risk-constrained framework is proposed in [17] for short-term optimal scheduling of autonomous microgrids to evaluate the influence of demand response (DR) programs on reliability and economic issues, simultaneously. Moreover, a two-stage stochastic programming has been proposed in [18] for joint DA energy and reserve scheduling, where the role of DR resources in the provision of required reserves to accommodate wind power deviation is discussed. A stochastic decision making strategy has been reported in [19] to coordinate the operation of WPP and DR aggregators (DRAs) taking part in DA market. In order to deal with uncertainties of wind power and market prices, conditional value at risk $(\mathrm{CVaR})$ has been included in the model. A regret-based stochastic bi-level framework for optimal decision making of a DR aggregator is addressed in [20] to purchase energy from short term electricity market and wind generation units is proposed. In addition, in [21], an optimal offering strategy has been presented for a WPP in a competitive environment in which the preferences of responsive loads are considered. In that study, the problem is formulated from the WPP viewpoint as a stochastic bi-level programming model to determine optimal bids and offering

Table I. The contribution of literatures in in view of existing state of the art.

\begin{tabular}{|c|c|c|c|c|c|c|c|}
\hline Reference & Responsive load & $\mathrm{P} 2 \mathrm{P}$ & Bi-level & $\begin{array}{l}\text { energy and reserve } \\
\text { scheduling }\end{array}$ & Risk version & $\begin{array}{l}\text { Competition } \\
\text { among WPPs }\end{array}$ & Decision maker \\
\hline [4] & - & - & $\checkmark$ & - & - & - & WPP \\
\hline$[5]$ & - & - & - & - & $\checkmark$ & - & WPP \\
\hline [8] & - & - & $\checkmark$ & - & $\checkmark$ & - & WPP \\
\hline [9] & - & - & $\checkmark$ & $\checkmark$ & - & - & Storage system \\
\hline [10] & $\checkmark$ & - & - & $\checkmark$ & $\checkmark$ & - & Hybrid power plant \\
\hline [13] & - & - & - & $\checkmark$ & $\checkmark$ & - & WPP \\
\hline$[16]$ & $\checkmark$ & - & - & $\checkmark$ & $\checkmark$ & - & Microgrid operator \\
\hline [17] & $\checkmark$ & - & - & $\checkmark$ & $\checkmark$ & - & Microgrid operator \\
\hline [18] & $\checkmark$ & - & - & $\checkmark$ & $\checkmark$ & - & System operator \\
\hline [19] & $\checkmark$ & - & $\checkmark$ & - & $\checkmark$ & - & WPP \\
\hline [20] & $\checkmark$ & - & $\checkmark$ & - & $\checkmark$ & $\checkmark$ & DR aggregator \\
\hline [21] & $\checkmark$ & - & $\checkmark$ & - & $\checkmark$ & $\checkmark$ & WPP \\
\hline$[23]$ & - & $\checkmark$ & - & - & $\checkmark$ & - & Prosumers \\
\hline [24] & - & $\checkmark$ & - & - & - & - & Proposing a model \\
\hline$[27]$ & - & - & $\checkmark$ & - & $\checkmark$ & $\checkmark$ & Retailer \\
\hline [28] & $\checkmark$ & - & $\checkmark$ & - & $\checkmark$ & $\checkmark$ & DR aggregators \\
\hline This paper & $\checkmark$ & $\checkmark$ & $\checkmark$ & $\checkmark$ & $\checkmark$ & $\checkmark$ & WPP \\
\hline
\end{tabular}


prices, however, reserve procurement from DR participants is not considered.

Recently, peer-to-peer (P2P) electricity trading in distribution systems has been explored in order to properly manage existing prosumers and to continually promote a deeper penetration of prosumers Error! Reference source not found.. A P2P market structure based on a multi-bilateral economic dispatch formulation is proposed in [24], allowing for multi-bilateral trading with product differentiation also based on consumer preferences. The interconnection of smart homes that are considered as nanogrids in a microgrid can provide P2P energy trading among the households as explained in [25]. The research on P2P energy trading is still at an early stage and there is no consensus on what type of data sharing and processing infrastructure is more efficient and yields the best results [23]. In this context, due to the interactive and conflicting nature of energy trading among prosumers, P2P strategy is an effective mechanism for modeling the decision-making processes that needs to be more investigated. Table I is added to show the contributions of the recent works in view of existing state of the art literature.

In this study, a risk-averse stochastic decision making strategy is developed from the WPP's viewpoint based on a bi-level stochastic programming model. In this model, the WPP competes against the rivals to attract DRAs in order to maximize its profit, but also to minimize the DRAs' costs. The main contribution of this work is the presentation of a new method to manage $\mathrm{P} 2 \mathrm{P}$ in a wholesale market to cope with the direct interaction and negotiation among WPPs and DRAs. In P2P electricity trading, WPPs and DRAs are able to directly trade energy and reserve with each other to achieve a win-win outcome so that the buyers can save costs while the sellers can achieve a profit. Therefore, in the proposed structure, the WPP is able to purchase reserve from responsive loads to offset part of its wind power deviation and bid energy in the DA market. In order to incorporate the risk associated with the decisions of the WPP and to assess the effect of scheduling the reserve capacity, CVaR measure is employed in the proposed model. Therefore, the main novel contributions of this work are summarized in the following:

- A bi-level stochastic programming formulation is proposed to maximize WPP's profit in a competitive market. Accordingly, the WPP participates in DA and balancing markets while providing a part of its required reserve directly from DRAs, to manage the risk of wind power uncertainties.

- A P2P market structure is presented for trading energy and reserve between a WPP and DRAs, while the interaction of the WPP with the upstream network in the wholesale market is also considered.

- The impacts of wind forecast errors and DR participants on the WPP's decisions are investigated in different cases with and without implementing P2P mechanism in the reserve scheduling.

The rest of the paper is organized as follows. Section II explains the proposed decision making strategy. In Section III, the proposed approach is formulated as a bi-level stochastic optimization problem. Numerical results are presented and discussed in Section IV. Finally, conclusions of this study are drawn in Section V.

\section{FrameWORK OF THE PROPOSED DeCision MAKING MODEL}

The proposed decision making framework of the WPP in a competitive environment is depicted in Fig.1. In this structure, on one side, the WPP as a price-taker, participates in wholesale market and submits energy bids in DA market and compensates the energy deviations in the balancing market.

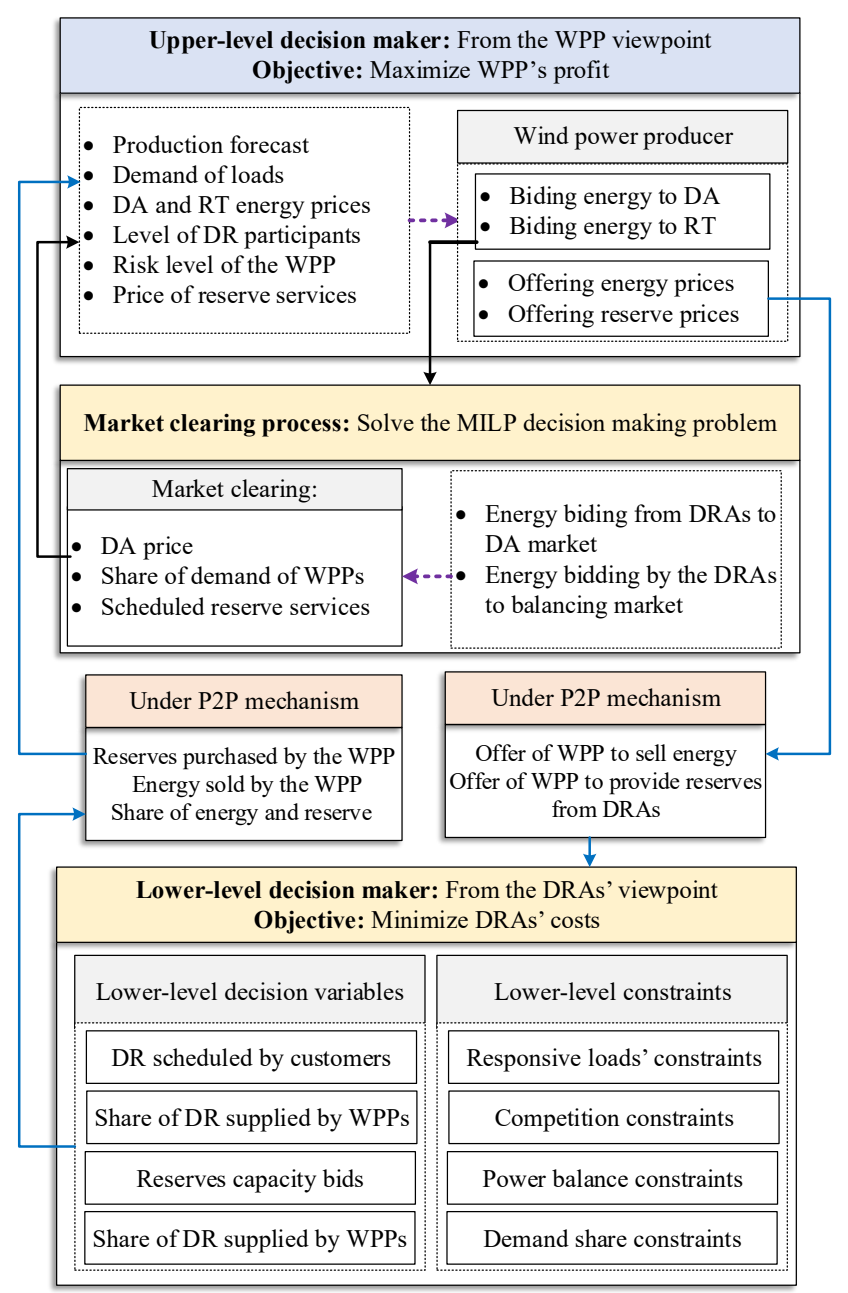

Fig. 1. Framework of decision making strategy of the WPP.

On the other side, at the retail level, the WPP competes against other WPPs to attract DRAs to supply their electricity demand in a P2P mechanism to maximize its expected profit. In fact, under a P2P electricity trading mechanism, the WPP offers energy price based on real time pricing (RTP) to the DRAs and is able to purchase the reserve capacity from them to offset a part of its wind energy deviations. The deployment of a two-way communication enables the interaction between the WPP and DRAs for energy-management schemes. Here, it is assumed that the customers under the jurisdiction of DRAs are equipped with intelligent energy controllers. So, the DRAs can respond to the prices by adjusting responsive demands. Moreover, the WPP can purchase some of its required reserve from DRAs to offset its energy deviation in the balancing market. On the other hand, each DRA can monitor the offering price of WPPs and supply the responsive demands under its jurisdiction from the most competitive WPP. This can be achieved by fast communication media developments with the ability of bidirectional data transfer 
among the DRAs and smart loads [21]. Therefore, as it can be seen in Fig.1, the proposed framework consists of a bi-level optimization problem, in which optimal bidding and offering strategies of the under study WPP are modeled in the upperlevel problem, which is the leader.

In the lower-level problem, which is the follower, the DA energy and reserve scheduling from DRAs' viewpoint are handled, so that their expected procurement costs are minimized. Therefore, the decision variables of the leader problem consist of purchasing/selling energy bids in DA and balancing markets, offering energy and reserve prices to DRA as well as reserve provided by the aggregators. In addition, the decision variables of the follower problem include the load scheduled by aggregators, the share of demand supplied by each WPP and the reserve provided by DRAs.

In this paper, in a $\mathrm{P} 2 \mathrm{P}$ trading floor, there are autonomous agents consisting of WPP and DRAs. Under a P2P trading framework, the WPP and DRAs are able to directly trade energy and reserve with each other to achieve a win-win outcome so that the WPP can obtain a higher profit while the DRAs can reduce their payments.

\section{Mathematical Model of The Proposed Bi-LeVEL PROBLEM}

In this section, the bi-level optimization framework that is composed of the bidding/offering strategy of the WPP in the upper-level and of the optimal scheduling of DRAs in the lower-level is mathematically modeled. The procedure to obtain the single level mixed-integer linear programming (MILP) problem is as follows:

- Replace the lower level problem with its KarushKuhn-Tucker (KKT) optimality conditions [22],

- Replace the bilinear products with their equivalent linear expressions using duality theory.

With transforming the bi-level problem to a single one, based on the scenarios generated for the DA and balancing market prices, the energy trading of the WPP with both DA and balancing market would be obtained. Also, the offering price by the WPP to the DRAs and the share of the WPP to supply the loads would be achieved. In fact, the WPP can obtain that based on the market prices, how much power it needs to trade with the wholesale market. Also, the WPP can achieve its share when competes against other rival WPPs to attract the DRAs.

The upper level and lower level are represented with their mathematical models in the next section. Each of the constraints of the lower level problem is followed by its respective dual variables separated by a colon.

\section{A. Model of the Upper-level Problem}

In the upper-level problem, the WPP seeks to maximize its expected profit by solving objective function as in (1).

$$
\begin{array}{r}
\operatorname{Max} \sum_{\omega \in \Omega} \pi_{\omega} \sum_{t \in T}\left[\begin{array}{l}
E_{t, \omega}^{D A, \text { Sell }} \lambda_{t, \omega}^{D A, \text { Sell }}-E_{t, \omega}^{D A, B u y} \lambda_{t, \omega}^{D A, B u y} \\
+E_{t, \omega}^{B^{+}} \lambda_{t, \omega}^{B^{+}}-E_{t, \omega}^{B^{-}} \lambda_{t, \omega}^{B^{-}} \\
+E_{t, \omega}^{W} \lambda_{t}^{W p p_{0}} \\
-\left(R_{t, \omega}^{u p} \lambda_{t}^{C_{u p}}+R_{t, \omega}^{d n} \lambda_{t}^{C_{d n}}\right) \\
+\left(-R_{t, \omega}^{u p} \lambda_{t, \omega}^{R_{u p}}+R_{t, \omega}^{d n} \lambda_{t, \omega}^{R_{d n}}\right) \rho_{t, \omega}^{d e l} \\
+\left(R_{t, \omega}^{u p} \lambda_{t, \omega}^{R_{u p}}+R_{t, \omega}^{d n} \lambda_{t, \omega}^{R_{d n}}\right) \rho_{t, \omega}^{d e l} \rho_{t, \omega}^{F O R}
\end{array}\right] \\
+\beta\left(\xi-\frac{1}{1-\alpha} \sum_{\omega=1}^{\Omega} \pi_{\omega} \eta_{\omega}\right)
\end{array}
$$

The first term of the objective function stands for the revenue/cost from selling/buying energy to/from the DA market, the second term presents the costs due to the positive/negative energy imbalance, the third line represents the revenue from selling energy to DRAs. The fourth line expresses the costs of up and down reserve capacity allocated by DRAs. The costs related to the real deployment of up and down reserves are provided in the fifth line and the sixth line represents the income earned from DRA for not providing up and down reserve when it is called upon to deploy reserve. In fact, in the last term, if DRA cannot deploy the contracted reserve when they are called, they should pay the cost as a penalty due to the un-deployment of reserve. Therefore, this term is considered as an income for the objective function of the WPP. Finally, the cost associated with risk management is represented by $\mathrm{CVaR}$ term multiplied by a weighting risk factor $\beta$, that is added to the objective function. This term allows the WPP to manage the degree of risk-aversion to cope with uncertainties. This optimization is subject to the following constraints.

The power generated from wind farms and the trading energy with the DA and balancing market as well as the reserve provided by the DRAs should equal to the load supplied by the WPP.

$$
\begin{aligned}
& E_{t, \omega}^{\text {wind }}-E_{t, \omega}^{D A, \text { Sell }}+E_{t, \omega}^{D A, B u y}-E_{t, \omega}^{B^{+}}+E_{t, \omega}^{B^{-}}+R_{t, \omega}^{u p}-R_{t, \omega}^{d n} \\
& =E_{t, \omega}^{W}+E_{t, \omega}^{N R L} / N_{W p p}
\end{aligned}
$$

The amount of energy that the WPP can sell and buy in DA and balancing markets are limited as below:

$$
\begin{aligned}
& 0 \leq E_{t, \omega}^{D A, \text { Sell/Buy }} \leq \bar{P}^{\text {Sell/Buy }} \\
& 0 \leq E_{t, \omega}^{B^{+/-}} \leq \bar{P}^{B^{+/-}}
\end{aligned}
$$

The constraints related to $\mathrm{CVaR}$ term are described as in equations (5) and (6):

$$
\sum_{\omega \in \Omega} \pi_{\omega} \sum_{t \in T}\left[\begin{array}{l}
E_{t, \omega}^{D A, \text { Sell }} \lambda_{t, \omega}^{D A, \text { Sell }}-E_{t, \omega}^{D A, B u y} \lambda_{t, \omega}^{D A, B u y} \\
+E_{t, \omega}^{B^{+}} \lambda_{t, \omega}^{B^{+}}-E_{t, \omega}^{B^{-}} \lambda_{t, \omega}^{B^{-}} \\
+E_{t, \omega}^{W} \lambda_{t}^{W p p_{0}} \\
-\left(R_{t, \omega}^{u p} \lambda_{t}^{C_{u p}}+R_{t, \omega}^{d n} \lambda_{t}^{C_{d n}}\right) \\
+\left(-R_{t, \omega}^{u p} \lambda_{t}^{R_{u p}}+R_{t, \omega}^{d n} \lambda_{t}^{R_{d n}}\right) \rho_{t, \omega}^{d e l} \\
+\left(R_{t, \omega}^{u p} \lambda_{t}^{R_{u p}}+R_{t, \omega}^{d n} \lambda_{t}^{R_{d n}}\right) \rho_{t, \omega}^{d e l} \rho_{t, \omega}^{F O R}
\end{array}\right]
$$

$\eta_{\omega} \geq 0$ 
The inclusion of the weighted factor $\beta$ underlines the balance between risk and expected profit.

The WPP estimates its share to supply demand of loads with the following constraint [27]:

$$
E_{t, \omega}^{W}=E_{t, \omega}^{D} \sum_{\phi \in \Phi} \pi_{\phi} X_{t, \phi}^{W p p_{0}}
$$

\section{B. Model of the Lower-level Problem}

In the lower-level problem, the DRAs participate in DA energy and provide reserve services to the WPP in P2P trading floor such that to minimize their payment costs. Thus, the objective function of this level can be written as follow:

$$
\operatorname{Min}\left[\begin{array}{c}
\hat{E}_{t}^{D}\left[\lambda_{t}^{W p p_{0}} X_{t, \phi}^{W p p_{0}}+\sum_{\substack{W p p \in N_{W p p} \\
W p p \neq W p p_{0}}} \lambda_{t, \phi}^{W p p} X_{t, \phi}^{W p p}\right] \\
\sum_{W p p \in N_{W p p}} \sum_{W p p^{p} \in N_{W p p}} \hat{E}_{t}^{D} \lambda_{W p p, W p p^{F}}^{F} Y_{t, \phi}^{W p p, W p p^{\prime}} \\
-\left(R_{t, \omega}^{u p} \lambda_{t}^{C_{u p}}+R_{t, \omega}^{d n} \lambda_{t}^{C_{d n}}\right)+\left(-R_{t, \omega}^{u p} \lambda_{t, \omega}^{R_{u p}}+R_{t, \omega}^{d n} \lambda_{t, \omega}^{R_{d n}}\right) \rho_{t, \omega}^{d e l} \\
+\left(R_{t, \omega}^{u p} \lambda_{t, \omega}^{R_{u p}}+R_{t, \omega}^{d n} \lambda_{t, \omega}^{R_{d n}}\right) \rho_{t, \omega}^{d e l} \rho_{t, \omega}^{F O R}
\end{array}\right]
$$

The first line of the objective function represents the costs for purchasing energy from both the WPP under study and rival WPPs. The second line expresses switching cost for hesitation of the DRAs to switch among WPPs. The third line represents the income due to up and down reserve allocated and deployed by DRAs. Since the relation in (8) represents the cost minimization from the DRA perspective in the lower level of the problem, the income parts are given with "-." sign. The last line states the penalty cost for DRA, if it does not deploy reserve when it is called.

The constraints related with the lower level problem are given as follows:

$$
\begin{aligned}
& X_{t, \phi}^{W p p}=X_{W p p, t, \phi}^{\text {Init }} \\
& +\sum_{\substack{W p p \in N_{W p p} \\
W p p \neq W p p^{\prime}}} Y_{t, \phi}^{W p p, W p p^{\prime}}-\sum_{\substack{W p p^{\prime} \in N_{W p p} \\
W p p^{\prime} \neq W p p}} Y_{t, \phi}^{W p p^{\prime}, W p p}: K 1_{\phi}
\end{aligned}
$$

Constraint (9) represents the shift of demand of DRAs among WPPs and constraint (10) explains that each DRA should be connected to only one WPP to supply its demand [28].

$$
X_{t, \phi}^{W p p_{0}}+\sum_{\substack{W p p \in N_{W p p} \\ W p p \neq W p p_{0}}} X_{t, \phi}^{W p p}=100 \%: K 2_{\phi}
$$

The up and down reserves provided by the DRAs are restricted by constraints (11) and Error! Reference source not found.).

$$
0 \leq R_{t, \omega}^{d n / u p} \leq E_{t, \omega}^{D}: K 3_{t, \omega}: K 6_{t, \omega}
$$

\section{Solution to the Proposed Bi-level Model}

Fig. 2 illustrates the flowchart of the proposed solution methodology for decision making of the WPP. In the first step, Monte Carlo simulations (MCSs) and Roulette wheel mechanism (RWM) strategies are employed to generate random scenarios of different uncertainties by using their associated probability density functions (PDFs) [21].
Then, the generated scenarios are reduced to a limited set by using K-means procedure as an appropriate scenarioreduction algorithm [29]. The reduced scenarios are applied to the proposed bi-level optimization model that is solved by replacing the constraints of follower problem with KKT optimality conditions and duality theory. In this regard, the upper level and the lower-level are incorporated using Lagrangian equation of the lower level problem. The components of the Lagrangian are the lower level objective function (8), equality constraints (9) and (10) and the inequality constraint of (11) as explained briefly in [30]. Also, the bilinear product of terms are obtained using strong duality theorem [31]. Finally, the resulting single-level MILP problem transformed to standard form, can be solved using commercially available branch-and-cut software [32]-[33].

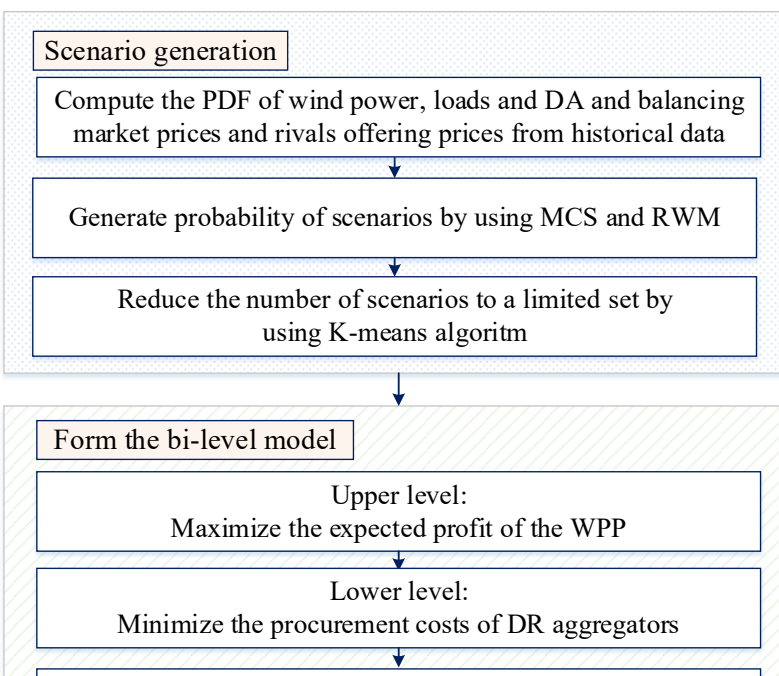

Transform the bi-level stochastic to a single one using KKT optimality conditions

Replace the nonlinear terms with the linear expressions using strong duality theory

Solve the MILP model of the obtained single level stochastic
decision-making problem

The DR aggregators receive the hourly energy prices and reserve capacity accepted by the WPP

The DR aggregators determine their hourly loads and reserves capacity for the next day and submit them to the WPP

The bidding decisions of WPP and reserve quantity are updated in balancing market

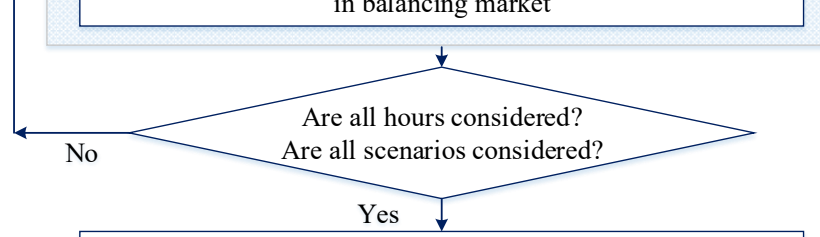

Compute the expected profit of the WPP, CVaR, share of each

WPPs in supplying loads, scheduling energy and reserves of DR aggregators, trading energy in DA and balancing markets

Fig. 2. Flowchart of the proposed solution methodology of the problem. 


\section{CASE STUDY AND NUMERICAL RESULTS}

\section{A. Case Study and Input Data}

The proposed bi-level model is implemented on realistic data from Nordic market [34], to determine optimal decisions of a WPP (i.e., $\mathrm{WPP}_{0}$ ) in competitive condition with the presence of other rivals (i.e., $\mathrm{WPP}_{1}, \mathrm{WPP}_{2}$ and $\mathrm{WPP}_{3}$ ). The predicted demand and wind output power of $\mathrm{WPP}_{0}$ are considered as shown in Fig. 3. Moreover, the forecasted values of DA and balancing market prices are shown in Fig. 4 , which are extracted from Nord pool market in October, 2018 [34]. The scenarios associated with forecasting errors of wind, DA and balancing market prices as well as rivals' offering prices are produced by mean and standard deviation based on the PDFs [31]. Simulations were run using CPLEX 12.6.0.0 under GAMS 24.2.2 [33] on a Dell Precision laptop with an Intel Core ${ }^{\mathrm{TM}} \mathrm{i}$ @ $2.6 \mathrm{GHz}$ processor and $16 \mathrm{~GB}$ of RAM. It should be noted that with considering a mip gap of $0 \%$, the computation time for the studied cases was between 3 and 6 minutes, with an average of 4 min and 42 seconds.

\section{B. Numerical Results and Discussions}

In the competitive environment, the WPP competes with rival WPPs and offers energy and reserve prices through a P2P mechanism in order to motivate DRAs to purchase their required energy from it and also sell their reserve capacity to it. The prices offered by the WPP obtained from the program together with the rivals' forecasted prices are shown in Fig. 5. Here, it is assumed that the WPP has accessibility to the historical data of rivals' prices and forecasts their offering energy and reserve prices for its decision making. Moreover, rate of load participant in DR is considered $40 \%$ and risk aversion parameter is fixed at 0.01 . As observed, the WPP often tries to offer middle prices to compete with rivals in order to maximize its revenue. The optimal decisions of the WPP are impressed by the DA market price signal, rivals' offering prices and wind and load data. To evaluate the impact of uncertainty of wind power on the optimal amount of energy and reserve capacity scheduled by the WPP, the simulation results for three levels of wind forecast error i.e. $5 \%, 10 \%$ and $15 \%$ are investigated.

Offering price signals of the WPP and its share to supply the demand of customers under a P2P contract in different levels of wind power uncertainty are depicted in Fig. 6. As mentioned earlier, in a competitive environment, the DRAs try to supply their required energy from the most competitive WPP. Therefore, during high price periods (for example during 8:00-11:00 and 18:00-22:00) the share of the WPP to supply demand of DRAs is relatively high. As observed from this figure, when wind power uncertainty augments, the WPP increases its offering prices in some periods to compensate its profit losses that occur in undesired scenarios.

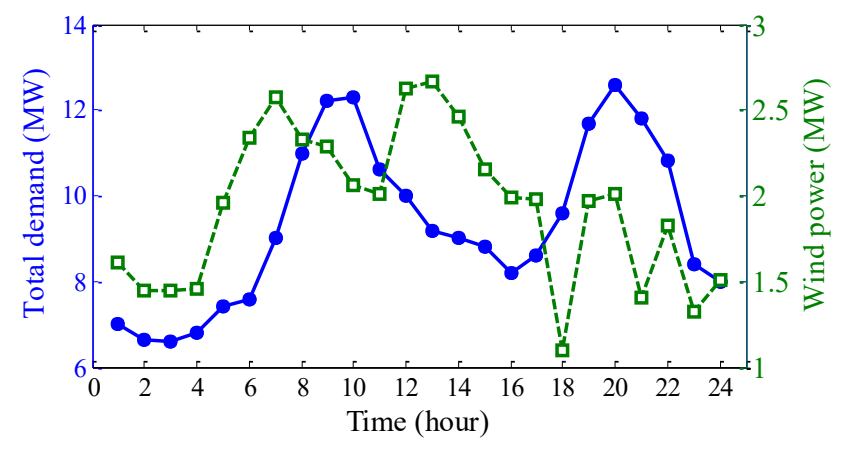

Fig. 3. The forecasted values of total load and wind output power of $\mathrm{WWP}_{0}$

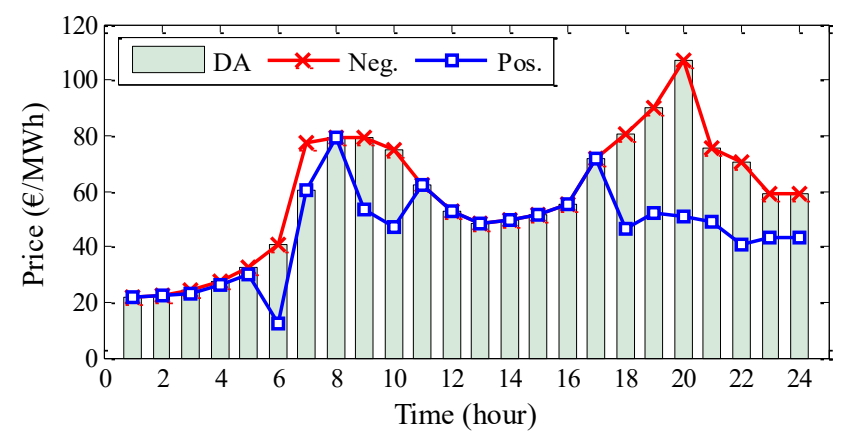

Fig. 4. Hourly electricity prices of DA and balancing market.

Fig. 7 shows the amount of buying/selling energy from/to the DA market for three mentioned wind forecast errors. Generally, the WPP tends to sell high energy quantities during high DA price periods and high wind energy production (i.e. 12:00-16:00). However, in some periods, although DA prices and wind production are high, the WPP

TABLE II

THE EFFECT OF WIND POWER UNCERTAINTY AND DR PARTICIPANTS ON WPP'S DECISIONS IN TWO CASES

\begin{tabular}{|c|c|c|c|c|c|c|c|c|c|}
\hline \multicolumn{10}{|c|}{ Joint energy and reserve scheduling under P2P mechanism } \\
\hline$\sigma_{\text {wind }}$ & Action & $\begin{array}{c}\text { Profit } \\
(€)\end{array}$ & $\begin{array}{c}\mathrm{CVaR} \\
(€) \\
\end{array}$ & $\begin{array}{c}\text { Up-reserve } \\
(\mathrm{MW})\end{array}$ & $\begin{array}{c}\text { Down-reserve } \\
(\mathrm{MW})\end{array}$ & $\begin{array}{c}\text { Total DA purchase } \\
\text { (MW) }\end{array}$ & $\begin{array}{c}\text { Total DA selling } \\
(\mathrm{MW})\end{array}$ & $\begin{array}{c}\text { Positive } \\
\text { (MW) }\end{array}$ & $\begin{array}{c}\text { Negative } \\
\text { (MW) }\end{array}$ \\
\hline \multirow{2}{*}{$5 \%$} & No DR & 2778.67 & -111.38 & - & - & 9.390 & 3.36 & 2.15 & 4.65 \\
\hline & With DR & 2956.26 & -30.013 & 4.726 & 0.065 & 11.559 & 8.86 & 2.17 & 5.04 \\
\hline \multirow{2}{*}{$10 \%$} & No DR & 2760.73 & -133.47 & - & - & 10.005 & 3.00 & 2.27 & 4.04 \\
\hline & With DR & 2940.08 & -37.69 & 4.825 & 0.235 & 12.282 & 8.08 & 2.32 & 4.02 \\
\hline \multirow{2}{*}{$15 \%$} & \begin{tabular}{|l|} 
No DR \\
\end{tabular} & 2493.23 & -160.47 & - & - & 14.730 & 0.02 & 3.61 & 2.90 \\
\hline & \begin{tabular}{|l|} 
With DR \\
\end{tabular} & 2785.89 & -55.41 & 5.177 & 0.916 & 13.938 & 3.94 & 3.41 & 2.95 \\
\hline \multicolumn{10}{|c|}{ Only energy scheduling } \\
\hline \multirow{2}{*}{$5 \%$} & No DR & 2801.36 & -112.50 & - & - & 9.619 & 3.37 & 2.08 & 4.29 \\
\hline & With DR & 2862.79 & -42.32 & - & - & 11.911 & 9.44 & 2.02 & 4.41 \\
\hline \multirow{2}{*}{$10 \%$} & \begin{tabular}{|l} 
No DR \\
\end{tabular} & 2747.57 & -134.24 & - & - & 9.919 & 2.91 & 2.42 & 4.11 \\
\hline & \begin{tabular}{|l|} 
With DR \\
\end{tabular} & 2839.64 & -49.77 & - & - & 12.181 & 9.13 & 2.44 & 4.11 \\
\hline \multirow{2}{*}{$15 \%$} & No DR & 1662.81 & -1389.28 & - & - & 19.144 & 0.07 & 3.87 & 3.29 \\
\hline & With DR & 2578.34 & -56.34 & - & - & 13.24 & 4.02 & 2.47 & 4.10 \\
\hline
\end{tabular}


purchases more energy to supply the customers under its jurisdiction (i.e. 8:00-12:00).

Also, the WPP purchased aggressively from DA market to supply high requested load during 18:00-22:00 due to lower wind generation. Moreover, as shown in this figure, purchased energy from DA market increases and sold energy decreases when wind power uncertainty augments. In fact, by increasing wind power uncertainty, the WPP tends to buy more energy from DA market and sell lower energy to decrease power deviations in the real-time.

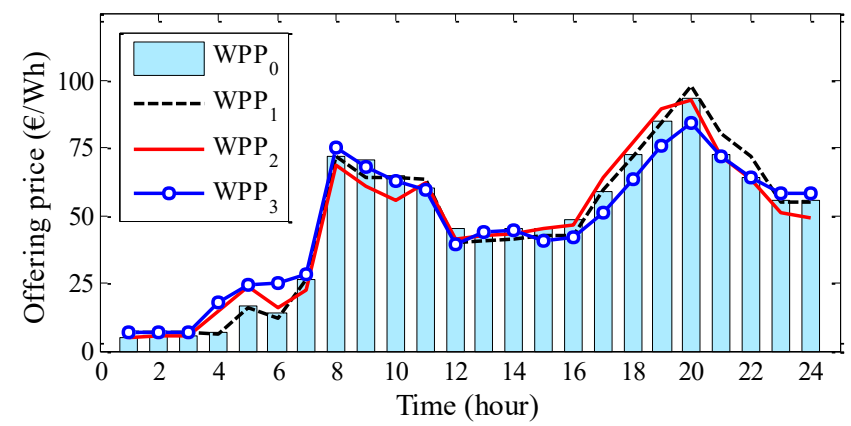

Fig. 5. Offering prices by WPPs during scheduling day.

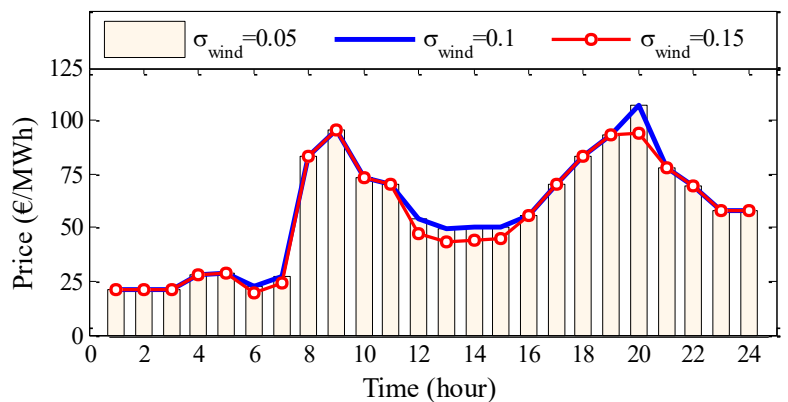

Fig. 6. Offering price by the under study WPP in different wind forecasted error.

As mentioned before, in order to compensate the energy mismatch and cover the energy deviations, the WPP participates in positive and negative balancing market as shown in Figs. 8 and 9, respectively. As it can be seen, when wind generation is low, the WPP compensates energy deficit in negative balancing market (e.g., during 19:00-22:00), while it sells surplus energy in positive balancing market when wind power production is high (e.g., during 12:0016:00). Moreover, by increasing wind power uncertainty, the WPP purchases less energy from negative balancing market and sells more energy to positive balancing market to compensate the probable energy mismatch.

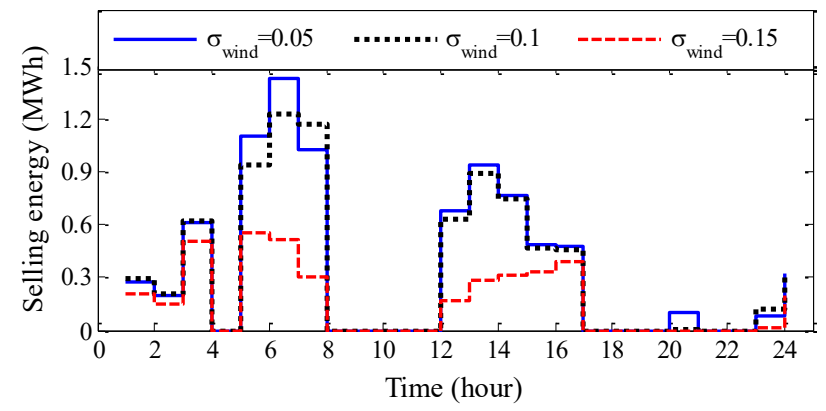

(a) DA energy selling

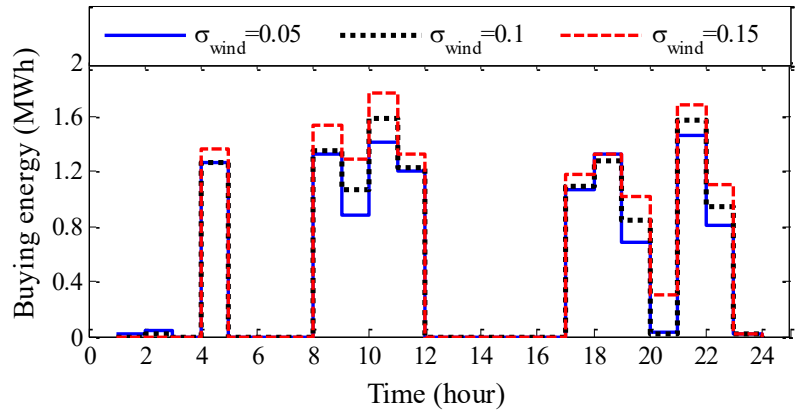

(b) DA energy buying

Fig. 7. Amount of buying/selling energy from/to the DA market.

In fact, when wind power has large deviation, the WPP tries to provide more reserve capacity from demand side recourses instead of purchasing extra high price energy. Therefore, by deploying reserve in the real-time, the amount of energy sold in balancing market increases and bought energy decreases.

Figs. 10 and 11 respectively depict up and down spinning reserves deployed by DRAs in P2P electricity trading to overcome the wind power uncertainty under different wind forecast error. As shown, when increasing wind power uncertainty, both up and down deployed reserve increases. The higher wind power uncertainty results in revenue losses for the WPP due to participating in costly balancing market. Therefore, providing reserve from DRAs brings opportunities for the WPP to increase its revenue compared with purchasing energy in the balancing market. In other words, in higher uncertainty, the WPP tends to purchase more reserve from DRAs to offset its output power deviation rather than fully taking part in the real time market. It should be noted that, when the WPP requests reserve services, DRAs curtail some of the responsive loads to cover the wind underprovision and increase their consumption to consume the wind over-provision. Moreover, the result shows that the pattern of up and down reserves follow the load profile supplied by the WPP.

The effect of wind power uncertainty as well as DR participants on the WPP's profit and energy trading in the two cases with and without providing reserve by DRAs is investigated and summarized in Table II. The results show that in the case with DR, when the WPP schedules for joint energy and reserve, its profit is higher than that when scheduling only for energy. Moreover, in both cases the participation of responsive loads under the jurisdiction of each aggregator in DR programs causes a better profit for the WPP compared to the case without DR.

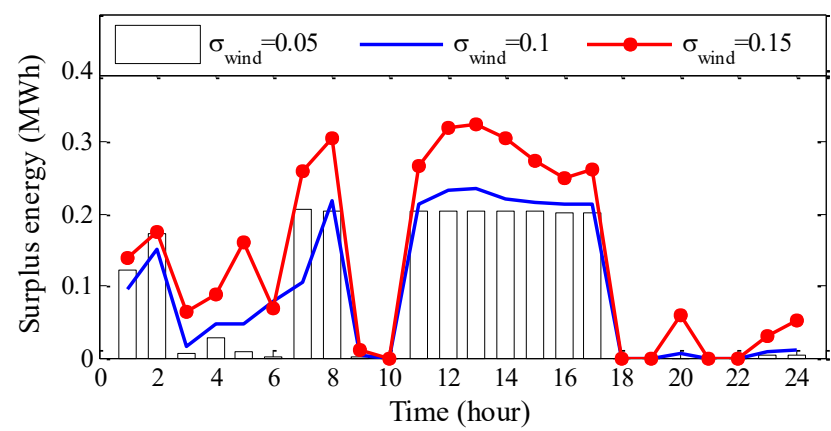


Fig. 8. Positive energy imbalance.

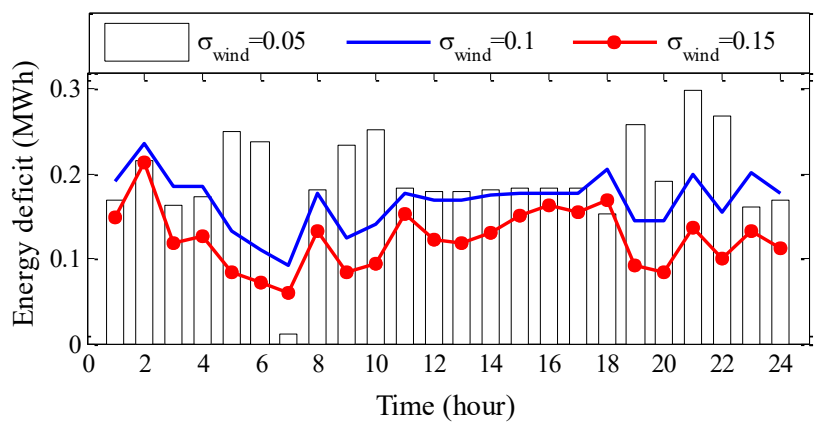

Fig. 9. Negative energy imbalance.

By participating in DR, responsive loads have the permission to choose their WPPs and the uncertainty of WPPs' output power is appropriately covered, and as a result, CVaR index augments severely by increasing DR participants. Moreover, by increasing wind uncertainty, customers provide more up and down reserves for the WPP to cover the wind power variability. In addition, in high level of wind power uncertainty, the WPP purchases more energy from DA market and sells lower energy to it. In addition, when increasing wind uncertainty, the WPP purchases more reserve to compensate the profit loss associated with wind power production uncertainty. Furthermore, when increasing wind uncertainty, $\mathrm{CVaR}$ index reduces, which indicates that there are some undesired profit scenarios which are far from the mean profit.

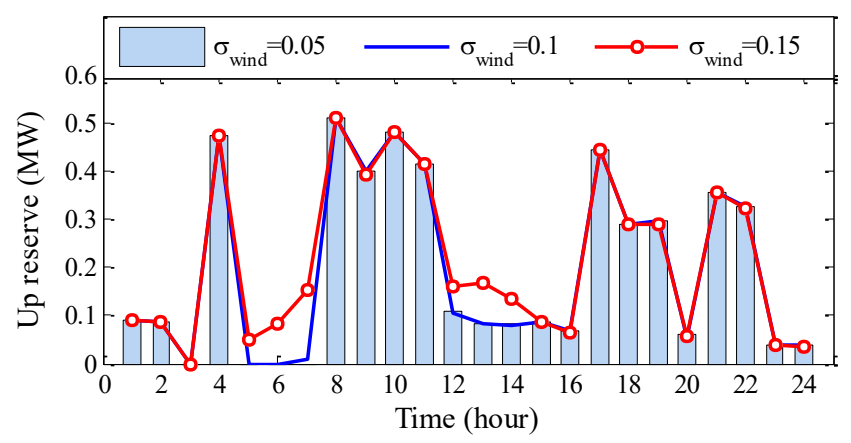

Fig. 10. Up reserve.

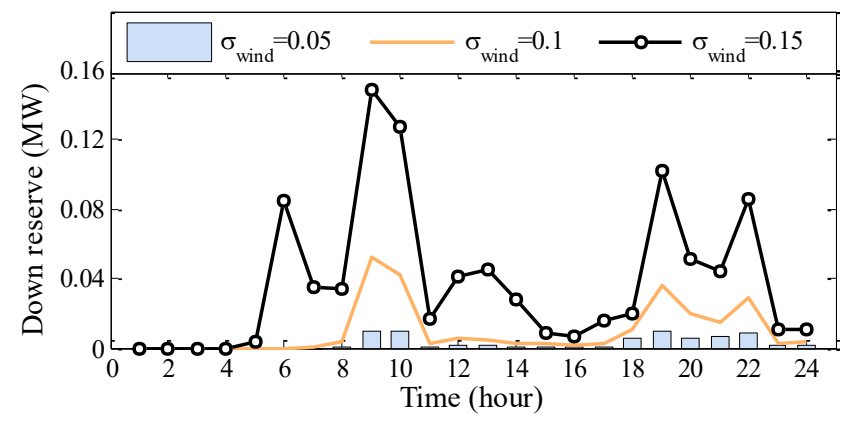

Fig. 11. Down reserve.

CVaR, expected profit, DA buying and selling values and the energy compensation in the balancing market as given in Table III. As seen, different items are provided in the two cases with and without DR. As shown, in both cases, with increasing $\beta$, the WPP confronts with lower expected profit and higher CVaR values. In fact, as the WPP behaves more risk aversely, it loses its profit, because, the profits that are far from the mean value would be eliminated. Negative values of CVaR in the case No DR indicates that the WPP may experience the profit losses in some scenarios. This implies the importance of risk management by the WPP as a decision maker.

As the WPP becomes more risk averse, its purchases and sells from/to the DA market reduces. In fact, as the WPP becomes more conservative, it tends to reduce its energy arbitrage with uncertain resources. Moreover, with increasing risk averse factor, the participation in positive balancing market decreases while the opposite happens for negative balancing market.

As more loads participate in DR programs, since the WPP would obtain more profit, CVaR values would become positive that indicates the WPP does not lose profit in some scenarios. Moreover, the energy exchange of the WPP with DA market augments. Because, the under study WPP can attract more loads in such a competitive environment that it should supply them. Also, as the loads become responsive, the uncertainties resulted from the behavior of the clients augments that should be compensated in the balancing market. So, the energy exchange in the balancing market augments.

\section{CONCLUSIONS}

This paper developed a decision making strategy for a WPP to determine optimal bid for energy in DA and balancing markets. In addition to energy scheduling, under a P2P mechanism, WPP purchased its required reserve from DRAs

Table III. Different items in different risk aversion factors in the cases without and with DR participants

\begin{tabular}{|c|c|c|c|c|c|c|c|c|c|c|c|c|}
\hline \multirow{2}{*}{$\beta$} & \multicolumn{2}{|c|}{ CVaR } & \multicolumn{2}{|c|}{ Expected profit } & \multicolumn{2}{|c|}{ DA buying } & \multicolumn{2}{|c|}{ DA selling } & \multicolumn{2}{|c|}{ Positive } & \multicolumn{2}{|c|}{ Negative } \\
\hline & No DR & With DR & No DR & With DR & No DR & With DR & No DR & With DR & No DR & With DR & No DR & With DR \\
\hline 0.01 & -160.47 & 1.72 & 2493.23 & 2969.33 & 14.730 & 15.783 & 0.020 & 8.688 & 3.612 & 3.545 & 2.905 & 3.125 \\
\hline 1 & -158.87 & 2.63 & 2492.99 & 2970.44 & 14.570 & 15.517 & 0.020 & 8.660 & 3.654 & 3.417 & 2.903 & 2.972 \\
\hline 5 & -158.87 & 2.81 & 2492.99 & 2969.96 & 14.570 & 15.526 & 0.020 & 8.582 & 3.654 & 3.452 & 2.903 & 2.925 \\
\hline 10 & -158.87 & 3.01 & 2492.99 & 2968.47 & 14.570 & 15.591 & 0.020 & 8.463 & 3.654 & 3.507 & 2.903 & 2.842 \\
\hline 15 & -158.87 & 3.10 & 2492.99 & 2967.36 & 14.570 & 15.666 & 0.020 & 8.431 & 3.654 & 3.680 & 2.903 & 2.943 \\
\hline 20 & -158.84 & 3.10 & 2492.51 & 2967.36 & 14.587 & 15.666 & 0.020 & 8.431 & 3.660 & 3.682 & 2.898 & 2.944 \\
\hline 100 & -158.84 & 3.10 & 2492.51 & 2967.36 & 14.587 & 15.666 & 0.020 & 8.431 & 3.660 & 3.547 & 2.898 & 2.809 \\
\hline 1000 & -158.84 & 3.109 & 2492.51 & 2967.36 & 14.587 & 15.666 & 0.020 & 8.431 & 3.660 & 3.547 & 2.898 & 2.809 \\
\hline
\end{tabular}


to reduce its revenue loss due to different uncertainty under competitive environment. A stochastic bi-level problem was formulated that allowed the WPP to schedule for joint energy and reserve to offset part of its output power deviation, instead of being penalized in balancing market. Therefore, the objective of the upper level of the problem was defined as the WPP's profit maximization while the objective in the lowerlevel minimized costs of DRAs. Moreover, CVaR approach was incorporated in the decision making model to tackle the uncertainty of the problem. The optimal decision strategy provided an optimal way for the WPP to manage its power deviation by scheduling some reserve from demand side resources. To verify the optimal decision making strategy, the proposed model was tested on a realistic case study and the results demonstrated that:

- when the WPP purchased reserves capacity from responsive loads through $\mathrm{P} 2 \mathrm{P}$ trading, its profit increased by $3.5 \%$ with respect to the case without considering reserve services;

- by increasing wind power uncertainty, the WPP scheduled for more up and down reserves to reduce the energy traded in the balancing market by deploying reserves in the realtime.

\section{REFERENCES}

[1] P. Siano, "Demand response and smart grids - A survey," Renew. Sust. Ener. Reviews, vol. 30, pp. 461-478, Feb. 2014.

[2] F. Bavafa, T. Niknam, R. Azizipanah-Abarghooee and V. Terzija, "A New Biobjective Probabilistic Risk-Based Wind-Thermal Unit Commitment Using Heuristic Techniques," IEEE Trans. Indus. Info., vol. 13, no. 1, pp. 115-124, Feb. 2017.

[3] A. Fabbri, T. G. San Roman, J. R. Abbad, and V. H. M. Quezada, "Assessment of the cost associated with wind generation prediction errors in a liberalized electricity market," IEEE Trans. Power Syst., vol. 20, no. 3, pp. 1440-1446, Aug. 2005.

[4] H. Hao, and L. Fangxing, "Bidding strategy for wind generation considering conventional generation and transmission constraints," $J$. Mod. Power Syst. Clean Energy, vol. 3, no. 1, pp. 51-62, 2015.

[5] J.M. Morales, A. J. Conejo, and J. Perez-Ruiz, "Short-term trading for a wind power producer," IEEE Trans Power Syst., vol. 25, no. 1, pp.554- 564, Feb. 2010.

[6] L. Exizidis, J. Kazempour, P. Pinson, Z. D. Grève, and F. Vallée, "Impact of Public Aggregate Wind Forecasts on Electricity Market Outcomes," IEEE Tr.. Sus. Ene, vol. 8, no. 4, pp. 1394 - 1405, 2017.

[7] C. J. Dent, J. W. Bialek, and B. F.Hobbs, "Opportunity cost bidding by wind generators in forward markets: Analytical results," IEEE Trans. Power Syst., vol. 26, no. 3, pp. 1600-1608, 2011.

[8] H. T. Nguyen and L. B. Le, "Sharing Profit from Joint Offering of a Group of Wind Power Producers in Day Ahead Markets," IEEE Trans. Sustain. Energy, vol. 9, no. 4, pp. 1921 - 1934, 2018.

[9] E. Nasrolahpour, J. Kazempour, H. R. Zareipour, and W. D. Rosehart, "A Bilevel Model for Participation of a Storage System in Energy and Reserve Markets," IEEE Trans. Sustain. Energy, vol. 9, no.2, pp.582-598, 2018.

[10] J. Aghaei, M. Barani, M. Shafie-Khah, A. A. S. Nieta, and J. P. S. Catalão, "Risk-constrained offering strategy for aggregated hybrid power plant including wind power producer and demand response provider," IEEE Trans. Sust. En., vol. 7, no. 2, pp. 513-525, 2016.

[11] S. R. Konda, L. K. Panwar, B. K. Panigrahi, and R. Kumar, "Investigating the Impact of Load Profile Attributes on Demand Response Exchange," IEEE Trans. Indus. Info., vol. 14, no. 4, pp. $1382-1391,2018$.

[12] M. H. K. Tushar, A. W. Zeineddine, and C. Assi, "Demand-Side Management by Regulating Charging and Discharging of the EV, ESS, and Utilizing Renewable Energy," IEEE Trans. Indus. Info., vol. 14, no. 1, pp. $117-126,2018$.
[13] E. Du, N. Zhang, C. Kang, B. Kroposki, H. Huan, M. Miao, and Q. Xia, "Managing wind power uncertainty through strategic reserves purchasing," IEEE Trans. Power Syst., vol. 32, no. 4, pp. 2547-2559, 2017.

[14] S. R. Konda, L. K. Panwar, B. K. Panigrahi, and R. Kumar, "Optimal Offering of Demand Response Aggregation Company (DRACO) in Price Based Energy and Reserve Market Participation," IEEE Trans. Indus. Info., vol. 14, no. 2, pp. 578 - 587, 2018.

[15] N. Mohammad, and Y. Mishra, "Coordination of wind generation and demand response to minimise operation cost in dayahead electricity markets using bi-level optimisation framework," IET Gen. Transm. Distrib., vol. 12, no. 16, pp. 3793-3802, 2018.

[16] M. Vahedipour-Dahraie, H. Rashidizadeh-Kermani, H.R. Najafi, A Anvari-Moghaddam, and J. M. Guerrero, "Stochastic security and risk-constrained scheduling for an autonomous microgrid with demand response and renewable energy Resources," IET Renew. Power Gener., 2017, Vol. 11 Iss. 14, pp. 1812-1821.

[17] M. Vahedipour-Dahraie, H. Rashidizadeh-Kermani, A. AnvariMoghaddam, and J. M. Guerrero, "Stochastic Risk-Constrained Scheduling of Renewable-Powered Autonomous Microgrids with Demand Response Actions: Reliability and Economic Implications," Early access and to be published in IEEE Trans on Industry Applications.

[18] N. G. Paterakis, M. Gibescu, A. G. Bakirtzis, and J. P. S. Catalão, “A Multi-Objective Optimization Approach to Risk-Constrained Energy and Reserve Procurement Using Demand Response," IEEE Trans. Power Syst., vol. 33, no. 4, pp. 3940 - 3954, 2018.

[19] M. Asensio, and J. Contreras, "Risk-Constrained Optimal Bidding Strategy for Pairing of Wind and Demand Response Resources," IEEE Trans. Smart Grid, vol. 8, no. 1, pp. 200-208, 2017.

[20] H. Rashidizadeh-Kermani, M. Vahedipour-Dahraie, M. Shafie-khah, P. Siano, "A Regret-based Stochastic Bi-level Framework for Scheduling of DR Aggregator under Uncertainties," Accepted and to be published in IEEE trans. Smart Grid.

[21] H. Rashidizadeh-Kermani, M. Vahedipour-Dahraie, M. Shafie-khah, and J. P. S. Catalão, "A bi-level risk-constrained offering strategy of a wind power producer considering demand side resources," International Journal of Electrical Power \& Energy Systems, vol. 104, pp. 562-574, 2019.

[22] H. Rashidizadeh-Kermani, M. Vahedipour-Dahraie, A. AnvariMoghaddam, and J. M. Guerrero, "A stochastic bi-level decisionmaking framework for a load-serving entity in day-ahead and balancing Markets," Int. Trans. Electr. Energ. Syst. 2019; e12109.

[23] S. Cui, Y-W. Wang, Y. Shi, J. Xiao, " An Efficient Peer-to-Peer Energy Sharing Framework for Numerous Community Prosumers," Accepted and to be published in IEEE Trans. Indus. Info. doi: 10.1109/TII.2019.2960802.

[24] E. Sorin, L. Bobo, and P. Pinson, "Consensus-based Approach to Peer-to-Peer Electricity Markets with Product Differentiation," IEEE Trans. Power Systems, vol. 34, no. 2, pp. 994 - 1004, 2019.

[25] B. Nordman, and K. Christensen, "Local power distribution with nanogrids," 2013 International green computing conference proceedings, p. 1-8, 2013.

[26] P. Siano, G. De Marco, A. Rolan, V. Loia, A Survey and Evaluation of the Potentials of Distributed Ledger Technology for Peer-to-Peer Transactive Energy Exchanges in Local Energy Markets, , IEEE Systems Journal, Vol. 13 , Issue: 3 pp. 3454-3466, 2019

[27] M. Carrión, J.M. Arroyo, and A.J. Conejo, "A Bilevel Stochastic Programming Approach for Retailer Futures Market Trading," IEEE Trans. Power Syst., vol. 24, no. 3, pp. 1446-1456, 2009.

[28] H. Rashidizadeh-Kermani, M. Vahedipour-Dahraie, M. Shafie-khah, and J. P. S. Catalão, "Stochastic programming model for scheduling demand response aggregators considering uncertain market prices and demands," International Journal of Electrical Power \& Energy Systems, vol. 113, pp. 528-538, 2019.

[29] D. Arthur, and S. Vassilvitskii, "K-means++: The advantages of careful seeding," in Proc. 18th Annu. ACM-SIAM Symp Discrete Algorithms, New Orleans, LA, USA, 2007, pp. 1027-1035.

[30] H. Rashidizadeh-Kermani, M. Vahedipour-Dahraie, A. AnvariMoghaddam, and J. M. Guerrero, "Stochastic risk-constrained decision making approach for a retailer in a competitive environment with flexible demand side resources," Int. Trans. Electr. Energ. Syst. vol. 9, no. 2, 2019. 
[31] H. Rashidizadeh-Kermani, M. Vahedipour-Dahraie, H.R. Najafi, A. Anvari-Moghaddam, J. M. Guerrero, "A stochastic bi-level scheduling approach for participation of EV aggregators in competitive electricity markets," Appl. Sci. vol. 7, no. 10, pp. 1-16, 2017.
[32] Y. Lin, Y. Ding, Y. Song, and C. Guo, "A Multi-State Model for Exploiting the Reserve Capability of Wind Power," IEEE Trans. Power Syst., vol. 33 , no. 3, pp. 3358 - 3372, 2018.

[33] General Algebraic Modeling System (GAMS), www.gams.com

[34] Nordic Electricity, www.nordpool.com 